\title{
Erratum: Quantifying Entanglement of Maximal Dimension in Bipartite Mixed States [Phys. Rev. Lett. 117, 190502 (2016)]
}

Gael Sentís, Christopher Eltschka, Otfried Gühne, Marcus Huber, and Jens Siewert

(Q) (Received 5 April 2019; published 26 April 2019)

DOI: 10.1103/PhysRevLett.122.169901

We report an error in Eq. (4) of our publication. The middle line, defining the state $\tilde{\rho}_{1}$, should read

$$
\tilde{\rho}_{1}=\frac{1}{d-1} \sum_{m=1}^{d-1}\left|\Psi_{d}^{(m)}\right\rangle\left\langle\Psi_{d}^{(m)}\right|,
$$

where $\left|\Psi_{d}^{(m)}\right\rangle:=(1 / \sqrt{d}) \sum_{j} \exp (i 2 \pi j m / d)|j j\rangle$ is a maximally entangled state with phase factors. The error does not affect any of the results of the paper.

We acknowledge C. Jebarathinam for pointing out the error. 